\title{
Comparative analysis of serological tests and fecal detection in the diagnosis of Mycobacterium avium subspecies paratuberculosis infection
}

\author{
Hong-Tae Park ${ }^{1}$, Seungmin Ha², Hyun-Eui Park ${ }^{1}$, Soojin Shim¹, Tai Young Hur², Han Sang Yoo ${ }^{1, *}$ \\ ${ }^{1}$ Department of Infectious Diseases, College of Veterinary Medicine, Seoul National University, Seoul 08826, Korea \\ ${ }^{2}$ Department of Animal Resources Development, National Institute of Animal Science, Rural Development Administration, \\ Cheonan 31000, Korea
}

\begin{abstract}
Johne's disease (JD) caused by Mycobacterium avium subspecies paratuberculosis (MAP) is a chronic, wasting infectious disease in ruminants that causes enormous economic losses to the dairy and beef cattle industries. The most effective way to eradicate JD is to detect infected individuals as early as possible and remove them from the herd. However, it is difficult to detect infected individuals early with the currently using diagnostic methods. Two serological diagnostic kits commercially used worldwide and a fecal detection test were compared using 298 serum samples and feces of cattle in this study to present an efficient diagnostic method. Although there was a high correlation between the 2 serological diagnostic kits $\left(\mathrm{R}^{2}=0.7473\right)$, kit A showed a higher serological positive rate. However, the correlation between fecal tests and serological diagnosis was very low. MAP was also detected in fecal tests in many serologically negative individuals. In the periodical diagnosis of JD, MAP was detected in the feces of only cows with the higher antibody titer to MAP. These results suggest that for effective eradication of JD, early detection of infected individuals by fecal tests together with the serological tests currently in use and by removal of infected individuals are needed.
\end{abstract}

Keywords: paratuberculosis, diagnosis, enzyme-linked immunosorbent assay, polymerase chain reaction

*Corresponding author

Han Sang Yoo

Department of Infectious Diseases, College of Veterinary Medicine, Seoul National

University, 1 Gwanak-ro, Gwanak-gu, Seoul 08826, Korea

Tel: $+82-2-880-1263$

Fax: +82-2-874-2738

E-mail: yoohs@snu.ac.kr

ORCID

Hong-Tae Park

https://orcid.org/0000-0003-3415-2339

Seungmin $\mathrm{Ha}$

https://orcid.org/0000-0002-5152-1979

Hyun-Eui Park

https://orcid.org/0000-0001-7000-1832

Soojin Shim

https://orcid.org/0000-0002-1769-0293

Tai Young Hur

https://orcid.org/0000-0003-3129-2942

Han Sang Yoo

https://orcid.org/0000-0003-0548-0835

Conflict of Interest

The authors declare no conflicts of interest.

Received: May 11, 2020

Revised: June 15, 2020

Accepted: July 9, 2020

\section{Introduction}

Paratuberculosis or Johne's disease (JD) is a chronic granulomatous infection of the intestinal tract of cattle and other domestic and wild ruminants caused by Mycobacterium avium subspecies paratuberculosis (MAP) [1-3]. The disease is economically significant and is difficult to control due to the chronicity of disease, the challenges in the diagnosis of infected animals, the lack of treatment options and, in some contexts, the problem of MAP in wildlife reservoirs. Because of the long incubation period (2-7 years) of MAP, most infected cattle (approximately 95\%) are considered to be in subclinical stages of the disease, with less than $5 \%$ of infected cattle displaying clinical signs of illness [3]. MAP infection is a welfare issue that costs the US economy US\$250 million/year due to decreased milk production, adverse effects on reproductive performance and increased culling rates [2,4-6]. Additionally, MAP appears to survive pasteurization and could enter the human food chain through dairy products, meat and untreated water supplies $[7,8]$. The fecal-oral route is a major infection route for most new infections [3]. Several studies have indicated that fetal infection of MAP depends on the severity of the infection of dam [3,9]. Most MAP infections occur in the early neonatal period and are often associated with calf sucking of manure-contaminated teats and udders when ingesting colostrum [10]. Additionally, multiple-use maternity pens can serve as focal points to spread the infection to neonates. MAP may be passed through the colostrum and milk of cattle in the later stage of infection $[11,12]$. However, it is widely accepted that resistance to MAP infection increases with age. Based on MAP bacterial shedding in feces and other signs, JD is classified into 4 stages: silent, subclinical, clinical and advanced $[3,13]$.

A range of diagnostic techniques have been used to detect MAP infection in cattle, but their performance can vary widely depending on the stage of MAP infection $[13,14]$. The diagnosis of infection in cattle depends in part on 
whether diagnostic efforts are aimed at detecting infection in an individual animal or a herd. US scientists recommended testing dairy and beef production for various purposes based on several diagnostic methods. In addition, more rigorous testing should be applied to all seed stocks. Diagnostic tests for JD can be classified into 2 categories: organism detection tests (bacterial culture, polymerase chain reaction [PCR] assay, etc.) and immune response tests (enzyme-linked immunosorbent assay [ELISA], interferon-g test, etc.) [3,1419]. Each test has its relative advantages and applications. Isolation of MAP cultures from the feces of suspected animals provides a definitive diagnosis of infections [15]. However, the method has several limitations, such as being expensive, time consuming, and having low sensitivity. Therefore, molecular and serological diagnostic methods were implemented. Although those methods have been used for the diagnosis of MAP infection, there are still some problems to overcome. The PCR assay with fecal samples is widely applied in the early stages of infection during the discharge of MAP into feces. However, this method also has some problems to be addressed when applied in the field because cows discharge bacteria intermittently and do not shed after a certain period of infection [13]. Serological test based on ELISA also has sensitivity problem. It has been accepted that the antibody level against MAP increases later stage of infection [20]. In addition, fluctuations of test results have frequently been observed in subclinically infected animals [21].

Therefore, this study was aimed to find a way to improve MAP diagnosis by providing data related to the effectiveness of diagnostic methods which are widely used in the livestock field through mutual comparison of the diagnostic methods mentioned above.

\section{Materials and Methods}

\section{Sample collection}

The diagnostic efficiency among the 2 serological tests and the fecal detection with real-time PCR were compared using the serum and fecal samples of cattle subjected to JD diagnosis in our laboratory from 2013 to 2019 . A total of 298 sera and 271 feces were selected for the comparison of each method. Fecal samples were collected individually from the rectum and transported to the laboratory in a $4^{\circ} \mathrm{C}$ container. All samples were kept in the $-70^{\circ} \mathrm{C}$ deep freezer until analysis.

\section{Fecal detection with real-time PCR}

From the fecal detection of MAP, 2 MAP-specific targets, IS900 and ISMap02, were detected in the DNA extracted from feces by real-time PCR, and samples positive for both targets were diagnosed as MAP infection. DNA was extracted from feces using a previous method described in our lab [22]. Primers for IS 900 and ISMap02 were used as previously described by Park et al. [22] and Sevilla et al. [23], respectively. Real-time PCR was performed in duplex using the TaqMan probe method, and the reaction mixture for the PCR was $1 \times$ Rotor-Gene Probe PCR master mix (Qiagen Inc., USA), $400 \mathrm{nM}$ forward and reverse primers, $100 \mathrm{nM}$ probe, and $4 \mu \mathrm{L}$ of template solution. The final amount of the mixture was set to $20 \mu \mathrm{L}$ with D.W. PCR conditions were as follows: a total of 45 cycles of initial denaturation at $95^{\circ} \mathrm{C}$ for $5 \mathrm{~min}$, annealing at $95^{\circ} \mathrm{C}$ for $15 \mathrm{sec}$, and $60^{\circ} \mathrm{C}$ for $1 \mathrm{~min}$.

\section{ELISA}

ELISA experiments were performed with 2 commercially available ELISA kits, kit A (Idexx Laboratories Inc., USA) and kit B (IDvet Innovative Diagnostics, France). Both diagnostic kits are indirect ELISAs based on preabsorption with the $M$. phlei antigen. However, the 2 kits showed differences in specificity and sensitivity. Both ELISAs were performed according to the manufacturer's instructions. The results were determined by measuring the absorbance at $450 \mathrm{~nm}$ for both kits and calculating the sample-to-positive $(\mathrm{S} / \mathrm{P})$ ratio. $\mathrm{S} / \mathrm{P}$ ratios higher than 55 and 70 in kits $\mathrm{A}$ and $\mathrm{B}$, respectively, were determined to be positive. The remaining patients were considered negative for MAP infection.

\section{Statistical analysis}

Statistical correlation analysis was performed on the ELISA results of the 2 kits. Direct comparison of $\mathrm{S} / \mathrm{P}$ ratio values between the 2 ELISA kits was performed using Pearson's correlation coefficient test. The agreement between the results from the 2 ELISA kits and the results from fecal detection with PCR were analyzed using Cohen's kappa coefficient test. When the correlation coefficient had a value of $0-0.2$, the correlation was determined to have a degree of agreement of slight; fair, up to $0.21-0.4$; moderate, up to $0.41-0.60$; substantial, up to $0.61-0.80$; and almost perfect, up to $0.81-1.0$.

\section{Results}

Two serological diagnostic kits that are currently commercially available around the world were compared, and 298 sera collected in Korea were analyzed for JD; the 2 kits showed a relatively high correlation $\left(\mathrm{R}^{2}=0.7473\right)$. The positive rates from kits $\mathrm{A}$ and $\mathrm{B}$ were $37.5 \%$ and $23.1 \%$, respectively. Therefore, kit A showed a higher detection rate of MAP infection than kit B (Fig. 1).

The correlation of the JD detection rate between the kit and fecal PCR was analyzed. The total number of positive and negative samples by kit A, kit B, and the fecal detection test with PCR from 298 samples is shown in Table 1. Additionally, Cohen's kappa coefficient analysis was performed to compare the concordance between each ELISA kit and fecal detection with PCR at the JD detection rate. As a result, no significant concordance in the detection rates was found between fecal detection with PCR and kit A (Cohen's kappa coefficient $=0.28, p>0.05$ ), while there was a very low correlation between fecal detection with PCR and kit B (Cohen's 


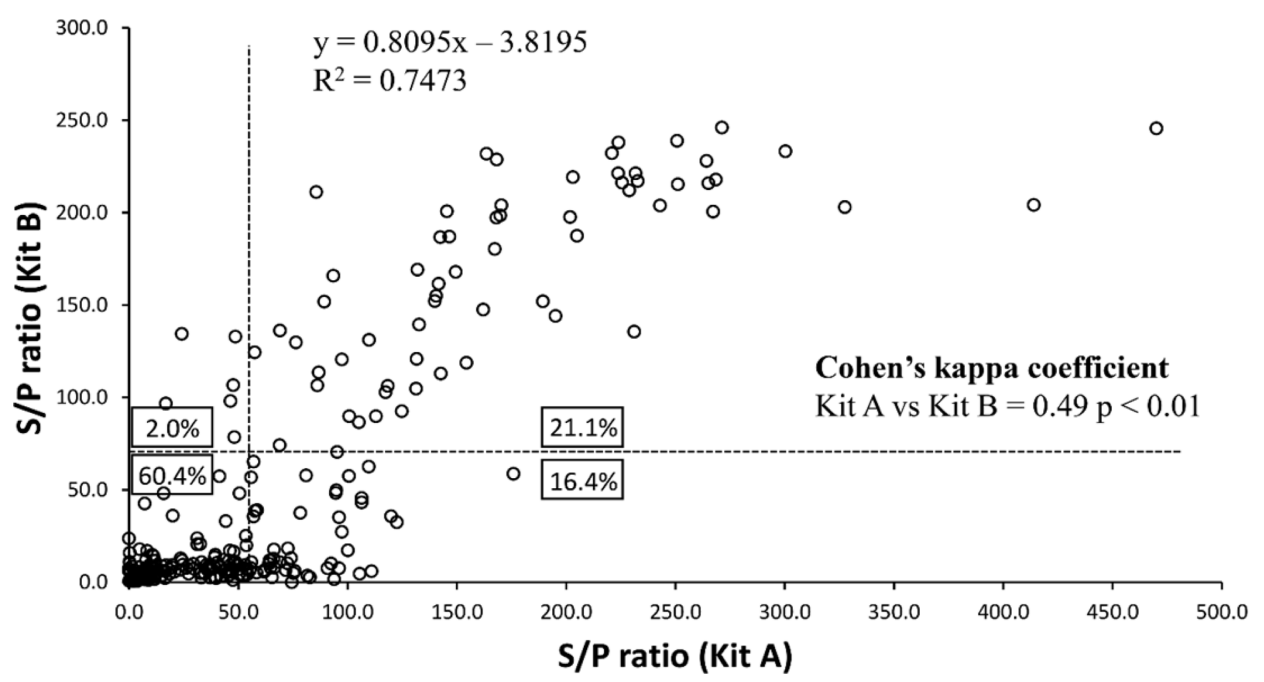

Fig. 1. Comparison of the serological detection rates by kits A and B. Individual serum samples subjected to diagnosis of Johne's disease were diagnosed with the 2 kits, and the positive and negative rates were compared. $\mathrm{S} / \mathrm{P}$, sample-to-positive.

Table 1. The total number of positive and negative samples by kit A, kit B, and the fecal detection test with PCR from 298 samples

\begin{tabular}{lccrrr}
\hline \hline \multirow{2}{*}{ Fecal PCR } & \multicolumn{2}{c}{ ELISA } & \multirow{2}{*}{ Total } \\
\cline { 2 - 5 } & Both positive & Kit A only & Kit B only & Both negative & 60 \\
Positive & 25 & 2 & 2 & 31 & 127 \\
Negative & 37 & 43 & 4 & 22 & 211 \\
ND & 1 & 4 & 0 & 180 & 27 \\
Total & 63 & 49 & 6 & 298 \\
\hline
\end{tabular}

PCR, polymerase chain reaction; ELISA, enzyme-linked immunosorbent assay; ND, not determined.

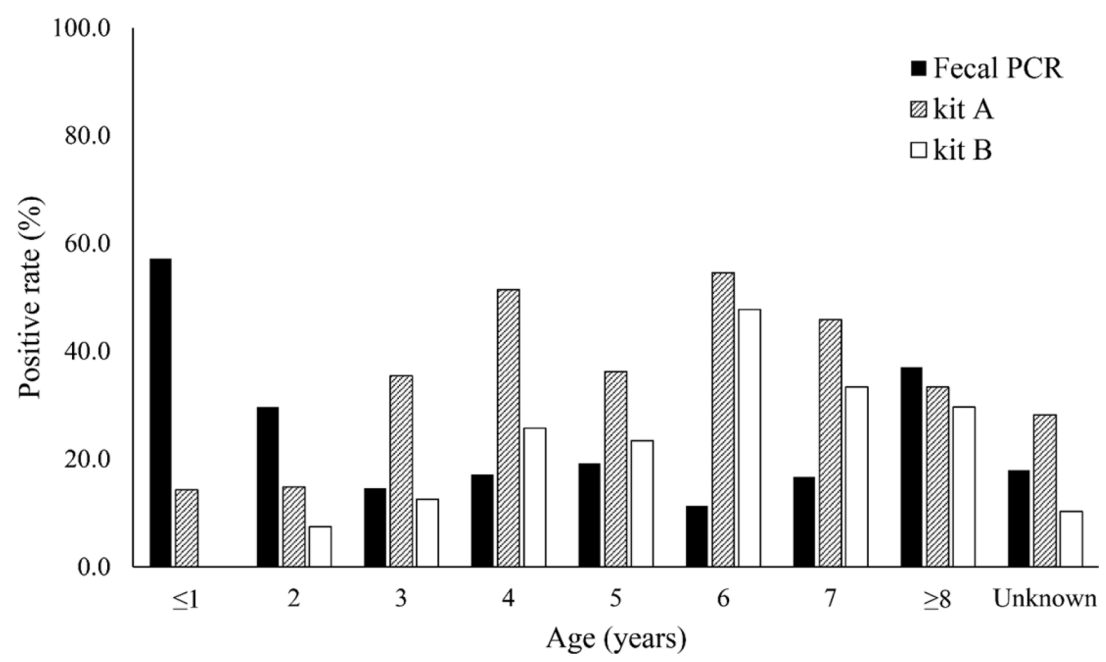

Fig. 2. Percentage of positives with serological tests, kits A and B, and fecal detection of Mycobacterium avium subspecies paratuberculosis with PCR by age.

$\mathrm{PCR}$, polymerase chain reaction.

kappa coefficient $=0.07, p<0.01)$. However, although the agreement was generally high (Cohen's kappa coefficient $=$ $0.30, p<0.01$ ) between fecal detection with PCR and serological tests when the samples were positive in both kits $A$ and $\mathrm{B}$, these results suggest that fecal detection with PCR and ELISA showed very low agreement.

In the analysis of serological and fecal PCR tests according to age, fecal PCR was effective until 2 years old, but 

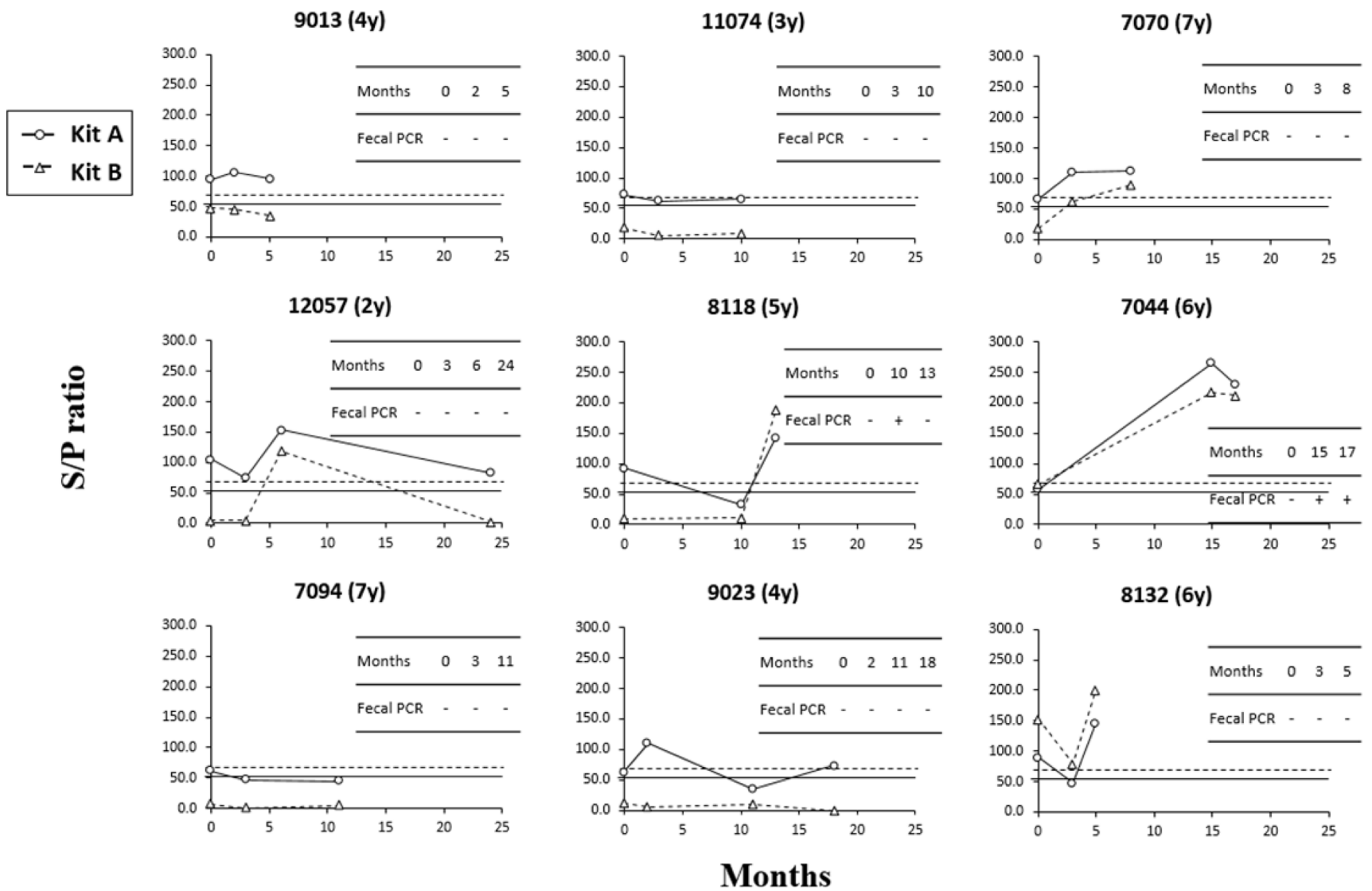

Fig. 3. Comparative analysis of individual trends using serological, kits $\mathrm{A}$ and $\mathrm{B}$, and fecal detection with polymerase chain reaction.

serological tests were more effective at 3 years of age or older (Fig. 2). In addition, kit A showed a higher detection rate than kit $\mathrm{B}$ at all ages (Fig. 2).

The diagnostic patterns of each ELISA kit and the PCR fecal test were compared in 20 cows that were tested 3 or more consecutively among all serum samples. The results in the 2 kits were completely consistent in the 11 samples, and the remaining 9 samples showed inconsistent results. The test results of the nine individuals showing inconsistent results are shown in Fig. 3. Although positive and negative diagnostic results differed depending on the kit, the change pattern by the $\mathrm{S} / \mathrm{P}$ ratio was very similar. In addition, the trend in the $\mathrm{S} / \mathrm{P}$ ratio showed that the $\mathrm{S} / \mathrm{P}$ values in kit $\mathrm{A}$ were higher than those in kit B, except for one (8132). These 2 observations indicated that the sensitivity of kit A seems to be higher, and this pattern was also seen in the results with 298 sera samples. However, in one individual (9023), the diagnosis result of kit A also went from positive to negative with time and did not show a tendency to coincide with the result of kit B. In that case, the fecal test with PCR has never been positive. Another thing to note is that the positive and negative results changed depending on the sampling time of the individual. Seven out of nine animals that showed different patterns in the diagnostic results were positive and negative in at least one kit, depending on the sampling time (Fig. 3).

\section{Discussion}

JD is a chronic, wasting disease, resulting in significant economic loss in dairy and beef herds in many countries due to chronic diarrhea, weight loss, progressive emaciation and death [1-3]. Therefore, the identification and removal of animals shedding the infectious agent before they become significant sources of contamination on a farm is the main measure of disease control at the herd level. The identification and removal of animals shedding the infectious agents before they become significant sources of contamination on a farm is the main measure of disease control at the herd level. Recently, 2 types of serological diagnostic kits have been made commercially available worldwide. However, they each have advantages and disadvantages in terms of specificity and sensitivity in field applications. Therefore, this study intends to propose a new suggestion for efficient control of MAP infection by comparing and analyzing 2 serological diagnostic kits currently used in Korea and fecal PCR.

The kit A showed a higher positive rate than kit $\mathrm{B}$ and showed only moderate agreement, despite the similar detection principle between the 2 kits. Because the specificity of 2 kits had been measured nearly $99.5 \%$ [24], it was considered that kit A have relatively higher sensitivity than kit B. This can also be supported by comparing the $\mathrm{S} / \mathrm{P}$ ratio values 
between the 2 kits, which are highly correlated with each other. Therefore, it would be suggested that kit A is suitable than kit B for the diagnosis of JD in Korea. However, the agreement of diagnostic results was relatively low compared to kappa values $(0.66$ to 0.91$)$ in previous studies [24,25]. It can be inferred that there are different situations between Korea and other countries. One possibility of inconsistency between previous and current results might be due to the difference of MAP strains prevalent in Korea and other countries. The causing of diagnostic bias between kit $\mathrm{A}$ and kit $\mathrm{B}$ should be proved in subsequent studies such as comparative genomic analysis of Korean MAP isolates.

The diagnostic efficiency of JD from fecal detection with PCR was compared with that from serological tests with kits $\mathrm{A}$ and $\mathrm{B}$, and the correlation between fecal PCR results according to serological diagnostic kits showed a similar pattern in kits $\mathrm{A}$ and $\mathrm{B}$. However, many of the positive individuals in the fecal PCR gave a negative serological test result. In comparison to fecal PCR and serological diagnostic tests according to age, the detection rates with fecal PCR were high in young and old groups, and coincidence rates with serological tests for the detection of MAP infection were high in the old age group. Although a high positive rate was serologically observed in 3- to 6-year-olds, the fecal PCR result had a low detection rate.

These results are thought to be closely related to the induction of the immune response following MAP infection in the pathogenesis of JD. In the early stage of MAP infection, cell mediated immune response which is characterized by activation of Th1 and Th17 $\mathrm{T}$ helper cells is occurred related to granuloma formation [26]. During this period, bacteria are excreted intermittently through the feces by the unknown factors. When the disease progresses, humoral immunity (Th2-derived immune response) becomes the main response against MAP infection [27]. During this period, increasing antibodies are frequently observed with increasing bacterial shedding [28]. However, it is not fully understood that which is the cause of immunological shift. Longitudinal study with experimental disease model showed that shedding of bacteria had seen in young calves (2-7 months post infection) and the increase of MAP specific antibody had been observed in later stage of infection only in a cow [29]. The authors also reported that bacterial shedding showed intermittent pattern.

The results from this study indicate that both serological diagnostic tests and fecal detection tests, which are currently used for the diagnosis of MAP infection, have complementary points. Therefore, to eradicate MAP infection in the herd, these 2 diagnostic methods should be applied efficiently according to age. In other words, the fecal test should mainly be performed at a young age, that is, until 2 years of age, and serological tests should mainly be performed at ages older than 3 years. Conclusively, to eradicate JD effectively, it is effective to detect infected individuals early by fecal PCR together with the serological tests currently in use and remove the infected individuals.

\section{Acknowledgements}

This work was carried out with the support of the Strategic Initiative for Microbiomes in Agriculture and Food (No. IPET918020-4), Agriculture, Food and Rural Affairs Convergence Technologies Program for Educating Creative Global Leader (No. 320005-4), Ministry of Agriculture, Food and Rural Affairs, Korea, the BK21 PLUS Program for Creative Veterinary Science Research, and the Research Institute for Veterinary Science, Seoul National University, Korea.

\section{References}

1. Manning EJ. Mycobacterium avium subspecies paratuberculosis: a review of current knowledge. J Zoo Wildl Med 2001;32:293-304.

2. Britton LE, Cassidy JP, O'Donovan J, Gordon SV, Markey B. Potential application of emerging diagnostic techniques to the diagnosis of bovine Johne's disease (paratuberculosis). Vet $\mathrm{J}$ 2016;209:32-39.

3. Fecteau ME. Paratuberculosis in Cattle. Vet Clin North Am Food Anim Pract 2018;34:209-222.

4. Kudahl AB, Nielsen SS, Østergaard S. Economy, efficacy, and feasibility of a risk-based control program against paratuberculosis. J Dairy Sci 2008;91:4599-4609.

5. Marcé C, Beaudeau F, Bareille N, Seegers H, Fourichon C. Higher non-return rate associated with Mycobacterium avium subspecies paratuberculosis infection at early stage in Holstein dairy cows. Theriogenology 2009;71:807-816.

6. Smith RL, Strawderman RL, Schukken YH, Wells SJ, Pradhan AK, Espejo LA, Whitlock RH, Van Kessel JS, Smith JM, Wolfgang DR, Gröhn YT. Effect of Johne's disease status on reproduction and culling in dairy cattle. J Dairy Sci 2010;93:3513-3524.

7. Grant IR, Ball HJ, Rowe MT. Incidence of Mycobacterium paratuberculosis in bulk raw and commercially pasteurized cows' milk from approved dairy processing establishments in the United Kingdom. Appl Environ Microbiol 2002;68:24282435.

8. Grant IR. Zoonotic potential of Mycobacterium avium ssp. paratuberculosis: the current position. J Appl Microbiol 2005; 98:1282-1293.

9. Aly SS, Thurmond MC. Evaluation of Mycobacterium avium subsp paratuberculosis infection of dairy cows attributable to infection status of the dam. J Am Vet Med Assoc 2005; 227:450-454.

10. National Research Council (US) Committee on Diagnosis and Control of Johne's Disease. Diagnosis and Control of Johne's Disease. Washington, D.C., National Academies Press, 2003.

11. Sweeney RW, Whitlock RH, Rosenberger AE. Mycobacterium paratuberculosis cultured from milk and supramammary lymph nodes of infected asymptomatic cows. J Clin Microbiol 1992;30:166-171.

12. Streeter RN, Hoffsis GF, Bech-Nielsen S, Shulaw WP, Rings DM. Isolation of Mycobacterium paratuberculosis from colostrum and milk of subclinically infected cows. Am J Vet Res 1995;56:1322-1324.

13. van Esker MH, Koets AP. Application of transcriptomics to enhance early diagnosis of Mycobacterium infections, with an 
emphasis on Mycobacterium avium ssp. paratuberculosis. Vet Sci 2019;6:59.

14. Arango-Sabogal JC, Fecteau G, Paré J, Roy JP, Labrecque O, Côté G, Wellemans V, Schiller I, Dendukuri N, Buczinski S. Estimating diagnostic accuracy of fecal culture in liquid media for the detection of Mycobacterium avium subsp. paratuberculosis infections in Québec dairy cows: a latent class model. Prev Vet Med 2018;160:26-34.

15. Prendergast DM, Pearce RA, Yearsley D, Ramovic E, Egan J. Evaluation of three commercial PCR kits for the direct detection of Mycobacterium avium subsp. paratuberculosis (MAP) in bovine faeces. Vet J 2018;241:52-57.

16. Buczinski S, Arsenault J, Kostoulas P, Corbière F, Fecteau G, Dendukuri N. Accuracy of paratuberculosis diagnostic tests in small ruminants: protocol for a systematic review and metaanalysis. Anim Health Res Rev 2019;20:98-102.

17. Gupta SK, Maclean PH, Ganesh S, Shu D, Buddle BM, Wedlock DN, Heiser A. Detection of microRNA in cattle serum and their potential use to diagnose severity of Johne's disease. J Dairy Sci 2018;101:10259-10270.

18. de Souza GD, Rodriguez AB, Romano MI, Ribeiro ES, Oelemann WM, da Rocha DG, da Silva WD, Lasunskaia EB. Identification of the Apa protein secreted by Mycobacterium avium subsp. paratuberculosis as a novel fecal biomarker for Johne's disease in cattle. Pathog Dis 2018;76:fty063.

19. Li L, Bannantine JP, Campo JJ, Randall A, Grohn YT, Schilling MA, Katani R, Radzio-Basu J, Easterling L, Kapur $\mathrm{V}$. Identification of sero-diagnostic antigens for the early diagnosis of Johne's disease using MAP protein microarrays. Sci Rep 2019;9:17573.

20. Nielsen SS, Toft N. Ante mortem diagnosis of paratuberculosis: a review of accuracies of ELISA, interferon- $\gamma$ assay and faecal culture techniques. Vet Microbiol 2008;129:217-235.

21. Facciuolo A, Kelton DF, Mutharia LM. Novel secreted antigens of Mycobacterium paratuberculosis as serodiagnostic biomarkers for Johne's disease in cattle. Clin Vaccine Immunol
2013;20:1783-1791.

22. Park HT, Shin MK, Sung KY, Park HE, Cho YI, Yoo HS Effective DNA extraction method to improve detection of Mycobacterium avium subsp. paratuberculosis in bovine feces. Korean J Vet Res 2014;54:55-57.

23. Sevilla IA, Garrido JM, Molina E, Geijo MV, Elguezabal N, Vázquez P, Juste RA. Development and evaluation of a novel multicopy-element-targeting triplex PCR for detection of Mycobacterium avium subsp. paratuberculosis in feces. Appl Environ Microbiol 2014;80:3757-3768.

24. Fry MP, Kruze J, Collins MT. Evaluation of four commercial enzyme-linked immunosorbent assays for the diagnosis of bovine paratuberculosis in Chilean dairy herds. J Vet Diagn Invest 2008;20:329-332.

25. Collins MT, Wells SJ, Petrini KR, Collins JE, Schultz RD, Whitlock RH. Evaluation of five antibody detection tests for diagnosis of bovine paratuberculosis. Clin Diagn Lab Immunol 2005;12:685-692.

26. DeKuiper JL, Coussens PM. Inflammatory Th17 responses to infection with Mycobacterium avium subspecies paratuberculosis (MAP) in cattle and their potential role in development of Johne's disease. Vet Immunol Immunopathol 2019;218:109954.

27. Manning EJ, Collins MT. Mycobacterium avium subsp. paratuberculosis: pathogen, pathogenesis and diagnosis. Rev Sci Tech 2001;20:133-150.

28. Koets A, Rutten V, Hoek A, van Mil F, Müller K, Bakker D, Gruys E, van Eden W. Progressive bovine paratuberculosis is associated with local loss of $\mathrm{CD}^{+} \mathrm{T}$ cells, increased frequency of gamma delta $\mathrm{T}$ cells, and related changes in $\mathrm{T}$ cell function. Infect Immun 2002;70:3856-3864.

29. Begg DJ, Plain KM, de Silva K, Gurung R, Gunn A, Purdie AC, Whittington RJ. Immunopathological changes and apparent recovery from infection revealed in cattle in an experimental model of Johne's disease using a lyophilised culture of Mycobacterium avium subspecies paratuberculosis. Vet Microbiol 2018;219:53-62. 